\title{
Plane and hemispherical potential structures in magnetically expanding plasmas
}

\author{
Kazunori Takahashi, ${ }^{a}$ Yuichi Igarashi, and Tamiya Fujiwara \\ Department of Electrical and Electronic Engineering, Iwate University, Morioka 020-8551, Japan
}

(Received 27 May 2010; accepted 6 July 2010; published online 28 July 2010)

\begin{abstract}
Two-dimensional potential structures are measured for different gas pressure in expanding argon plasma using permanent magnets, where the magnetic field is about $100 \mathrm{G}$ in the source and several gauss in the diffusion chamber. The plane potential drop is observed near the source exit for 0.35 mTorr, while the potential structure becomes hemispherical when increasing up to 1 mTorr; the hemispherical structure results in the radial divergence of the ion beam. It is found that the trajectories of the accelerated ions and the electrons overcoming the potential drop are dominated by the potential structure and magnetic-field lines, respectively. (C) 2010 American Institute of Physics. [doi:10.1063/1.3467857]
\end{abstract}

Expansion of low-pressure radiofrequency plasmas operated in inductively coupled or helicon wave mode along divergent magnetic-field lines has recently attracted a great deal of interests in communities of space and laboratory plasmas and electric propulsion because a nonlinear potential structure called an electric double layer (DL) spontaneously forms near the source exit and ions are subsequently accelerated by the potential drop. ${ }^{1}$ During the formation of the DL, the accelerated ion beam is electrically neutralized by electrons overcoming the potential drop. ${ }^{2}$ It has been suggested that this phenomenon can be utilized for development of an electrodeless plasma propulsion device. ${ }^{3}$ After the one-dimensional axial investigations in laboratory experiments, ${ }^{4-8}$ in analytical models, ${ }^{9-11}$ and in particle-incell simulation, ${ }^{12}$ the studies on the radial and/or twodimensional nature have been progressed in experiments ${ }^{13-19}$ for further development of the propulsion device, for development of two-dimensional analytical or simulation model, and for understanding its physics. In the series of the experiments on the magnetically expanding plasma using permanent magnets (PMs), it has been reported that the trajectory of the ion beam becomes spatially divergent when increasing the gas pressure from 0.35 to 1 mTorr. ${ }^{19}$ In contrast with the magnetically expanding plasmas, the hemispherical expansion in the "geometrically" expanding plasma has shown the generation of the strongly divergent ion beam, which is guessed to be due to the hemispherical plasma-potential structures. $^{20,21}$

In the present letter, we report a variation in the plasmapotential structure from a plane shape to a hemispherical shape when increasing the gas pressure in the magnetically expanding plasma, which is directly associated with the divergence of the ion beam. The results demonstrate the trajectory of the ions accelerated by the potential drop is dominated by the potential structure while it is found the electrons overcoming the potential drop move along the magnetic-field lines.

Experiments are performed in Permanent Magnets expanding Plasma machine at Iwate University (PMPI) shown in Fig. 1(a), which has been described previously. ${ }^{8}$ Briefly, the plasma source, consisting of a $20-\mathrm{cm}-$ long and $6.5-\mathrm{cm}-$

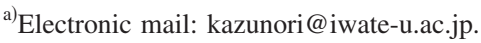

diameter glass tube surrounded by a double-turn loop antenna situated at $z=-9 \mathrm{~cm}$ and powered from an $\mathrm{rf}$ generator of $13.56 \mathrm{MHz}$ and $250 \mathrm{~W}$, is connected to a $30-\mathrm{cm}$-long and 26-cm-diameter grounded diffusion chamber, where $z=0$ is defined as the source exit. The chamber is evacuated to a base pressure of about $2 \times 10^{-6}$ Torr by a diffusion/rotary pumping system. The double concentric arrays of neodymium iron boron $(\mathrm{NdFeB})$ magnets surrounding the source tube provide an expanding magnetic field of about $100 \mathrm{G}$ in the source and a few gauss in the middle of the diffusion chamber as shown in Fig. 1(b). Argon gas is introduced from the source side and the gas pressure is maintained in the range of $0.35-1$ mTorr. The previous one-dimensional axial measurement has shown the rapid potential drop of the DL near the source exit over a few centimeters. ${ }^{8}$ The radial pro-

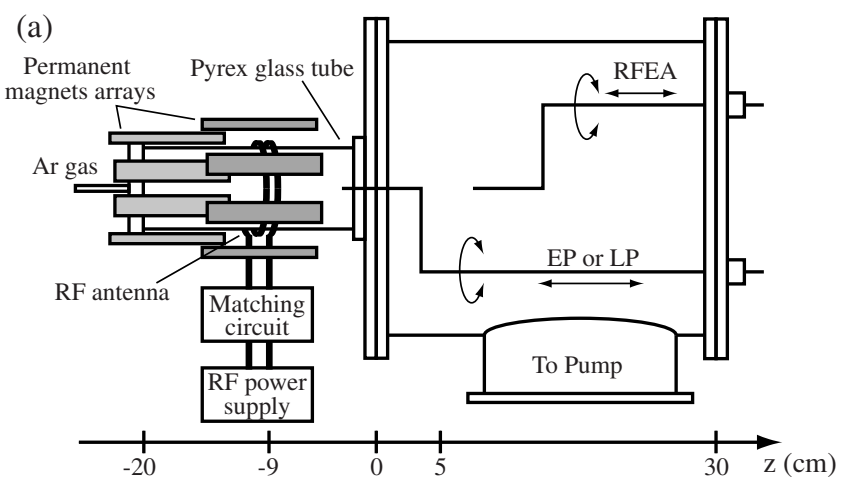

(b)

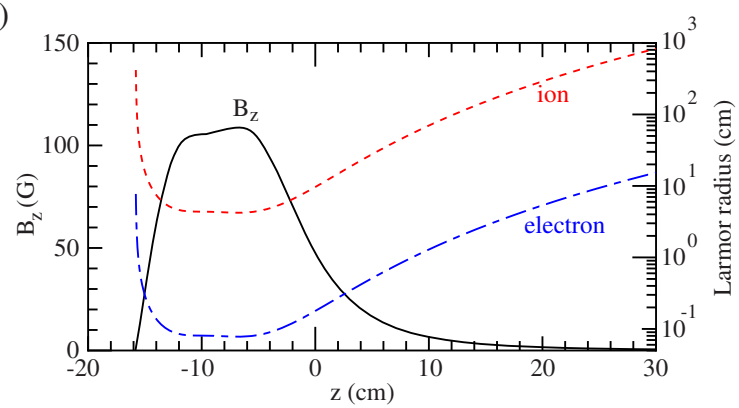

FIG. 1. (Color online) (a) Schematic diagram of PMPI. (b) Axial profile of the magnetic-field strength $B_{\mathrm{z}}$ (solid line), together with the calculated ion (dashed line) and electron (dotted-dashed line) Larmor radii, where the ion and electron temperatures are chosen as $0.2 \mathrm{eV}$ and $5 \mathrm{eV}$, respectively. 
(a) 0.35 mTorr

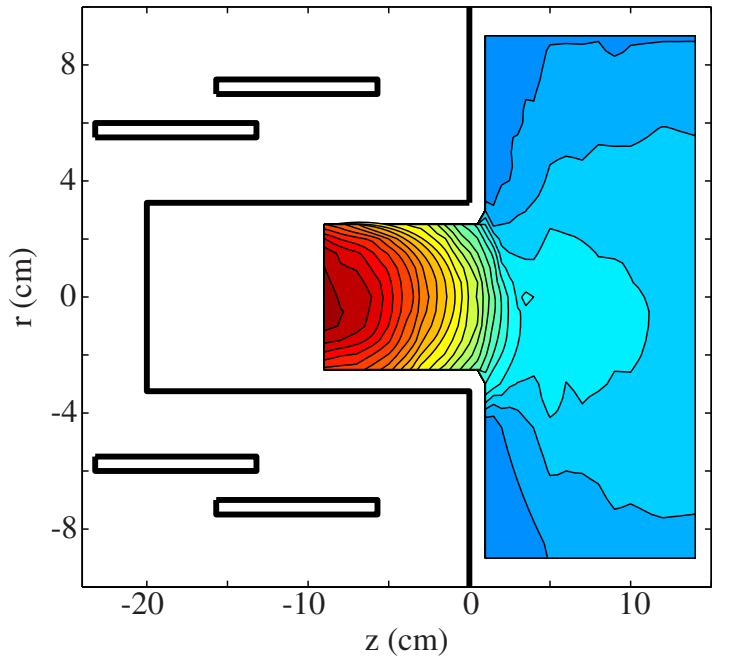

(b) 1 mTorr

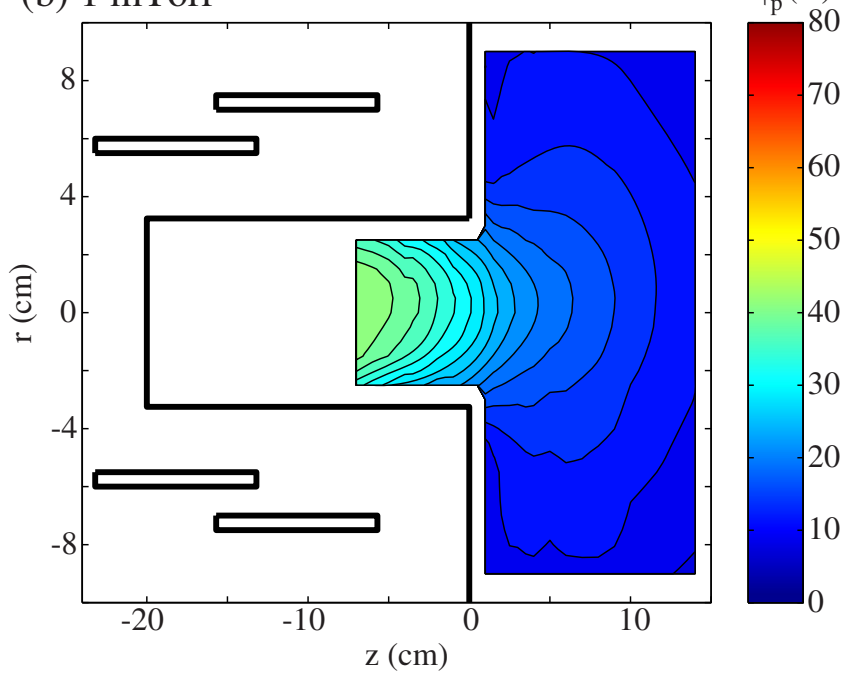

FIG. 2. (Color online) Two-dimensional $(r-z)$ contour plot of the local plasma potential $\phi_{\mathrm{p}}$ measured by the RFEA facing the radial wall for (a) $P_{\mathrm{Ar}}=0.35 \mathrm{mTorr}$ and (b) $P_{\mathrm{Ar}}=1 \mathrm{mTorr}$. The contour lines are drawn at 2.5 $\mathrm{V}$ intervals.

files of the ion beam current for various axial positions have already shown that the divergence of the beam for 0.35 mTorr is small while it becomes divergent when increasing the gas pressure up to 1 mTorr. ${ }^{19}$

Two-dimensional profiles of the local plasma potential $\phi_{\mathrm{p}}$ are measured by two different electrostatic probes of a retarding field energy analyzer (RFEA) and an emissive probe (EP). The RFEA facing the radial wall and the EP having support tubes bent as shown in Fig. 1(a), which are inserted from the downstream flange of the diffusion chamber, yield the approximate measurement in the $r-z$ plane by moving axially and rotating the probe shafts through the vacuum port. The Langmuir probe (LP) is also inserted instead of the EP for the measurement of the floating potential.

Figure 2 shows the contour plots of the local plasma potential $\phi_{\mathrm{p}}$ in the $r-z$ plane, measured by the RFEA facing radially, for (a) $P_{\mathrm{Ar}}=0.35 \mathrm{mTorr}$ and (b) $P_{\mathrm{Ar}}=1 \mathrm{mTorr}$, with the contour lines at $2.5 \mathrm{~V}$ intervals. We mention that the potential drop commonly depends on the gas pressure as observed in the magnetically expanding plasmas previously. ${ }^{9,22}$ The result for 0.35 mTorr in Fig. 2(a) shows the almost uniform potential profile in the diffusion chamber and the high potential in the source. Between these regions near the source exit, it is found that the plane-shape potential drop of the DL forms, where the collimated ion beam has been detected for the same conditions in the previous experiment. ${ }^{19}$ On the other hand, the result for 1 mTorr in Fig. 2(b) shows that the potential structure becomes hemispherical, where the strongly divergent ion beam has been observed for the same conditions in the previous experiment. ${ }^{19}$ In order to have more confidence on the variation from the plane to hemispherical structures, the potential measurements are performed by the EP (not shown here). The results do also show the very similar structures, i.e., the plane and hemispherical potential structures near the source exit for 0.35 mTorr and 1 mTorr, respectively. The potential structure has also been measured for stronger magnetic-field configuration, where the field strength in the source is increased up to about $270 \mathrm{G}^{22}$ The result shows the plane potential structure of the DL near the source exit, being similar to Fig. 2(a) (not shown here). For higher-pressure and low-magnetic-field conditions, the effects of the magnetic fields are reduced and the plasma becomes close to diffusive; then the situation becomes close to the geometrically expanding, no magnetic-field plasma. The previous work has already shown that the axial profiles of the geometrically expanding plasma can be fitted by the simple model assuming a spherical or hemispherical expansion. ${ }^{23}$ The ion flux conservation and the Boltzmann relation for electrons can create the hemispherical potential structures in this model. Therefore, it is considered that the Boltzmann electric field with hemispherical structure in the diffusion chamber is superimposed on the DL potential drop near the source exit for $1 \mathrm{mTorr}$ in the present experiment, where it is noted that the axial profile of the plasma potential for 1 mTorr in Ref. 19 has shown the gradual potential decrease in the diffusion chamber in addition to the rapid potential drop of the DL near the source exit.

For discussing the effect of the magnetic field on the ion beam trajectory, the calculated ion Larmor radius is plotted as dashed line in Fig. 1(b), where the ion temperature is chosen as $0.2 \mathrm{eV}$ (Ref. 24) for the calculation. The ion Larmor radius downstream of the source exit is found to be over $10 \mathrm{~cm}$, being much larger than the radial scale length of the potential structures; the ions are not restricted by the magnetic field lines and its trajectory would be dominated by the electric-field structure. Hence, we can deduce that the previously observed divergent ion beam for higher gas pressure of 1 mTorr originates in the hemispherical potential structure.

Figure 3(a) shows the calculated magnetic field lines produced by the PMs, where the dashed lines are the outermost field lines not intersecting the glass source wall (last field lines). In the previous one-dimensional, radial investigation of the electron energy distribution, the energetic electrons transported along the last field lines are detected. ${ }^{17,25}$ The easiest way to identify the presence of the energetic electrons is to look the floating potential $\phi_{\mathrm{f}}$ of the LP, where large negative potential against the local plasma potential would be an evidence of the energetic electrons. Figure 3(b) shows the radial profiles of $\phi_{\mathrm{f}}$ at $z=3,5$, and $7 \mathrm{~cm}$ for the gas pressure of 1 mTorr. The very clear narrow region of the large negative $\phi_{\mathrm{f}}$ is observed around $|r| \sim 5 \mathrm{~cm}$, and the region moves to radially outer side and expands in the radial direction as the probe moves toward the downstream, lowmagnetic-field side. It is found that these regions are in good 

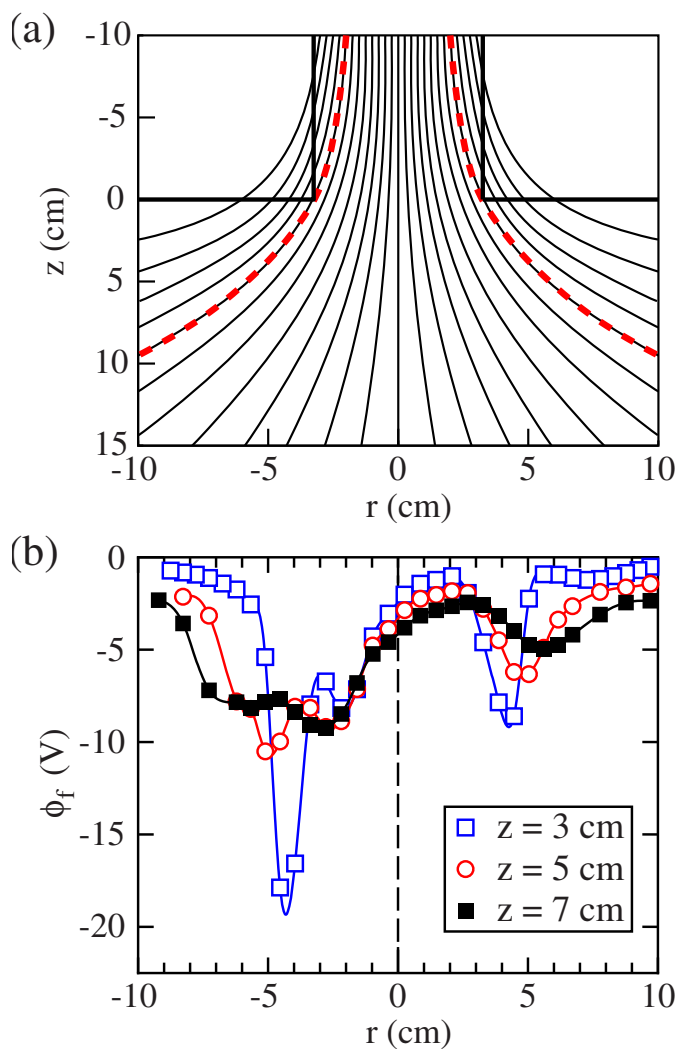

FIG. 3. (Color online) (a) Calculated magnetic-field lines in $r-z$ plane and the chamber geometry. (b) Radial profiles of the floating potential $\phi_{\mathrm{f}}$ of the LP for $1 \mathrm{mTorr}$ at $z=3 \mathrm{~cm}$ (open squares), $5 \mathrm{~cm}$ (open circles), and $7 \mathrm{~cm}$ (closed squares).

agreement with the position of the last field lines. The axial profile of the electron Larmor radius is plotted as dotteddashed line in Fig. 1(b), where the measured electron temperature of $5 \mathrm{eV}$ (Ref. 22) is used for the calculation. Since the electron Larmor radius is less than $1 \mathrm{~cm}$ at $z<10 \mathrm{~cm}$, the peripheral energetic electrons are restricted by the magnetic-field lines and are transported along the field lines. It is considered that the increase in the electron Larmor radius expands the narrow region with the energetic electrons in the downstream side as observed in Fig. 3(b). Therefore, it is found that the electron trajectory is dominated by the magnetic-field lines as contrasted to the ion trajectory decided by the potential structure.

In summary, the two-dimensional profiles of the plasma potential are measured in the magnetically expanding plasma using the PMs for two operating conditions of 0.35 and 1 mTorr. The plane potential drop formed for lower gas pressure of 0.35 mTorr can generate the spatially collimated ion beam while the structure is observed to become hemispherical and the ion beam becomes radially divergent when increasing the gas pressure up to 1 mTorr. Furthermore, the presence of the energetic electrons transported along the peripheral last magnetic-field lines is indicated. Due to the Larmor radius effects, it is found that the trajectory of the ions accelerated by the potential drop, i.e., the divergence of the ion beam, is dominated not by the magnetic field but by the potential structure while the electrons are restricted by and move along the magnetic-field lines.

The authors would like to thank Y. Shida and H. Chiba from Iwate University for their technical assistance, and Professor K. Takaki from Iwate University for helpful discussion. This work is partially supported by a Grant-in-Aid for Young Scientists (Grant Nos. B 20740317 and A 22684031) from the Ministry of Education, Culture, Sports, Science, and Technology, Japan. Part of this work is also supported by TEPCO Research Foundation, and by the Yazaki Memorial Foundation for Science and Technology.

${ }^{1}$ C. Charles, Plasma Sources Sci. Technol. 16, R1 (2007) and references therein.

${ }^{2}$ K. Takahashi, C. Charles, R. W. Boswell, T. Kaneko, and R. Hatakeyama, Phys. Plasmas 14, 114503 (2007).

${ }^{3}$ C. Charles, J. Phys. D 42, 163001 (2009) and references therein.

${ }^{4}$ X. Sun, S. A. Cohen, E. E. Scime, and M. Miah, Phys. Plasmas 12, 103509 (2005).

${ }^{5}$ C. Charles and R. W. Boswell, Phys. Plasmas 11, 1706 (2004).

${ }^{6}$ X. Sun, A. M. Keesee, C. Biloiu, E. E. Scime, A. Meige, C. Charles, and R. W. Boswell, Phys. Rev. Lett. 95, 025004 (2005).

${ }^{7}$ N. Plihon, P. Chabert, and C. S. Corr, Phys. Plasmas 14, 013506 (2007).

${ }^{8}$ K. Takahashi, K. Oguni, H. Yamada, and T. Fujiwara, Phys. Plasmas 15, 084501 (2008).

${ }^{9}$ M. A. Lieberman and C. Charles, Phys. Rev. Lett. 97, 045003 (2006).

${ }^{10}$ A. Fruchtman, Phys. Rev. Lett. 96, 065002 (2006).

${ }^{11}$ F. F. Chen, Phys. Plasmas 13, 034502 (2006).

${ }^{12}$ A. Meige, R. W. Boswell, C. Charles, and M. M. Turner, Phys. Plasmas 12, 052317 (2005).

${ }^{13}$ C. Charles, IEEE Trans. Plasma Sci. 33, 336 (2005).

${ }^{14}$ W. Cox, R. Hawkins, C. Charles, and R. W. Boswell, IEEE Trans. Plasma Sci. 36, 1386 (2008).

${ }^{15}$ W. Cox, C. Charles, R. W. Boswell, and R. Hawkins, Appl. Phys. Lett. 93, 071505 (2008).

${ }^{16}$ K. Takahashi, C. Charles, R. W. Boswell, and R. Hatakeyama, Phys. Plasmas 15, 074505 (2008)

${ }^{17}$ K. Takahashi, C. Charles, R. W. Boswell, W. Cox, and R. Hatakeyama, Appl. Phys. Lett. 94, 191503 (2009).

${ }^{18}$ C. Charles, R. W. Boswell, and R. Hawkins, Phys. Rev. Lett. 103, 095001 (2009).

${ }^{19}$ K. Takahashi and T. Fujiwara, Appl. Phys. Lett. 94, 061502 (2009).

${ }^{20}$ C. S. Corr, J. Zanger, R. W. Boswell, and C. Charles, Appl. Phys. Lett. 91, 241501 (2007).

${ }^{21}$ C. S. Corr, R. W. Boswell, C. Charles, and J. Zanger, Appl. Phys. Lett. 92, 221508 (2008).

${ }^{22}$ K. Takahashi, Y. Shida, and T. Fujiwara, Plasma Sources Sci. Technol. 19, 025004 (2010).

${ }^{23}$ C. Charles, R. W. Boswell, A. Bouchoule, C. Laure, and P. Ranson, J. Vac. Sci. Technol. A 9, 661 (1991).

${ }^{24}$ A. M. Keesee, E. E. Scime, C. Charles, A. Meige, and R. W. Boswell, Phys. Plasmas 12, 093502 (2005).

${ }^{25}$ C. Charles, Appl. Phys. Lett. 96, 051502 (2010). 